\title{
DANAE - a new experiment for direct dark matter detection with DEPFET silicon detectors
}

\author{
Hexi Shi ${ }^{* a}$ Alexander Bähr, ${ }^{b}$ Holger Kluck,,${ }^{a, c}$, Jelena Ninkovic, ${ }^{b}$ Jochen Schieck ${ }^{a, c}$ \\ and Johannes Treis ${ }^{b}$ \\ ${ }^{a}$ Institut für Hochenergiephysik der Österreichischen Akademie der Wissenschaften \\ Nikolsdorfer Gasse 18, 1050 Vienna, Austria \\ ${ }^{b}$ Max-Planck-Gesellschaft Halbleiterlabor \\ Otto Hahn Ring 6, 81739 Munich, Germany \\ ${ }^{c}$ Atominstitut, Technische Universität Wien \\ Stadionallee 2, 1020 Vienna, Austria \\ E-mail: Hexi.Shi@oeaw.ac.at
}

In the direct search for signals from the scattering of a dark matter particle candidate off standard model particles, the region of dark matter candidates with masses below $1 \mathrm{GeV} / \mathrm{c}^{2}$ remains mainly unexplored. We are preparing an experiment using a low-noise silicon detector as the sensitive target to search for signals from the inelastic scattering between a sub-GeV/c ${ }^{2}$ dark matter candidate and a bound electron in silicon. When such a scattering happens, energy deposit inside the silicon sensor of a few $\mathrm{eV}$ in the form of ionization can be identified using the low noise silicon detector applying the non-destructive repetitive readout method, which can be used to achieve an ENC of $0.2 e^{-}$rms and below.

PACS: Direct dark matter detection, Sub-GeV dark matter, DEPFET,

Silicon detector, Low-noise detector

ICHEP 2018, XXXIX International Conference on High Energy Physics

4-11 July 2018

Seoul, Republic of Korea

${ }^{*}$ Speaker. 


\section{Introduction}

Direct experimental searches of dark matter have been looking for signals from the elastic scattering between a relic dark matter particle and a nucleus [1]. Up to now, in the mass region between $1 \mathrm{GeV} / \mathrm{c}^{2}$ to $100 \mathrm{TeV} / \mathrm{c}^{2}$, no evidence has been found for the existence of such a particle. Experimental limitation in the sensitivity of direct searches towards sub-GeV/c dark matter comes firstly from the kinematics of the scattering. A $100 \mathrm{MeV} / \mathrm{c}^{2}$ dark matter candidate can create a nuclear recoil with a kinetic energy of a few eV. Taking into account the limits from the detectors, including the quenching factor and the intrinsic noise level of the detectors which determines the threshold of the signal, the nuclear recoil experiments can barely reach such a sensitivity.

Essig and others discussed in [2] the feasibility of detecting the energy deposit from a dark matter-electron recoil using low-noise semiconductor detectors as the active target to probe the mass region of dark matter even below the $\mathrm{MeV} / \mathrm{c}^{2}$ scale. Similar to the nuclear scattering, the energy deposit from a recoil electron is to be searched for in the form of an ionization signal. In a dark matter-electron scattering, which is an inelastic process, the kinetic energy of the dark matter must be large enough to excite a valence electron, which will further produce electron-hole pairs in the fully depleted silicon volume. This condition determines the minimal dark matter mass that can produce a detectable recoil signal to be at the order of $100 \mathrm{keV} / \mathrm{c}^{2}$. For dark matter candidates with masses in the $\mathrm{MeV} / \mathrm{c}^{2}$ to $\mathrm{GeV} / \mathrm{c}^{2}$ range, the estimated energy transfer to the recoil electron is a few $\mathrm{eV}$ to few tens of $\mathrm{eV}$, which correspond to one single to a few free electrons inside the silicon to be distinguished from the noise level in the charge collection and readout.

The pioneering application of a silicon device was done by the DAMIC experiment which used a Charge Coupled Device (CCD) with a noise level of a few electrons to search for ionization from dark matter induced nuclear recoils [3]. In order to improve the sensitivity towards the detection of electron recoil, the follow-up SENSEI experiment developed CCD arrays with non-destructive reptetitive readout (the skipper CCD), that achieved $\sim 0.1$ electron effective noise ${ }^{1}$ level [4] and will be used in long term physics run to search for dark matter-electron scattering events.

With different architecture and readout technique which excels in the readout speed at least 2-3 orders of magnitude faster than the CCD devices, the DEPFET (Deeply-depleted P-channel Field Effect Transistor) devices implementing the RNDR (Repetitive Non-Destructive Readout) architecture, can achieve a comparable readout noise level as the skipper CCD. The DEPFET devices allow us to search for dark matter particles with sub-GeV/c ${ }^{2}$ masses, which are accounted for by multiple theoretical models. The prototype of this detector has been developed by the Semiconductor Laboratory (HLL) of the Max Planck Society (MPG), and the performance of a single pixel has successfully reached the expected sub-electron noise level as reported in [5][6].

In the next sections, we will introduce the working concept of the DEPFET-RNDR based on the recent publication [5], and then present the status of the new project DANAE - $\underline{\text { Direct }} d \underline{\mathrm{A}} \mathrm{rk}$ matter detection using DEPFET with reptitive Non-destructive readout Application Experiment, that plans to apply this type of detector in the direct search of dark matter-electron recoils.

\footnotetext{
${ }^{1}$ When RNDR technique is applied, effective noise is defined as the standard deviation of the mean value of the repeated measurements for the amount of charge from a single time of charge collection.
} 


\section{DANAE experiment}

The goal of the DANAE experiment is to explore the sub-GeV/c ${ }^{2}$ mass region of the dark matter landscape, using the DEPFET detector as the active target. We will search for the events of dark matter bouncing off a bound electron inside the silicon volume. To identify the few $\mathrm{eV}$ energy deposit from the electron recoil, the two key requirements are the low-noise detector, and a low-background environment. Regarding the first requirement, one technology that stands out is the RNDR DEPFET, which is capable of reaching a sub-electron equivalent noise charge (ENC) in the readout [5][6], and hence became our choice of detection technique.

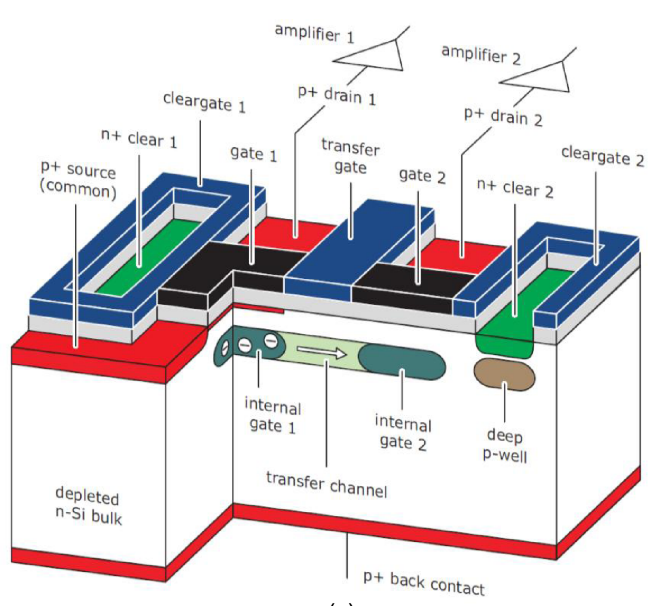

(a)

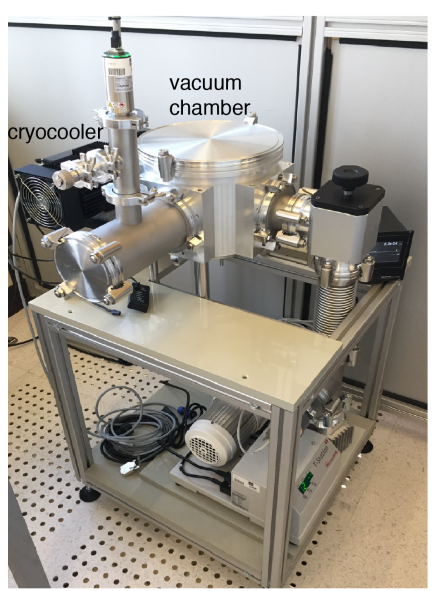

(b)

Figure 1: (a) illustration of a RNDR DEPFET "super-pixel", showing the elements to realize the RNDR; (b) the test setup at HLL, with a cooling system that will keep the DEPFET devices operating below $-70{ }^{\circ} \mathrm{C}$.

\subsection{DEPFET detector with RNDR}

The basic unit of a DEPFET-RNDR device, called a "super-pixel", as shown in Fig. 1 (a), was realized by a combination of two DEPFET devices [7] located on the same silicon bulk. Each of the two DEPFET devices consists of a p-channel-MOSFET (Metal-Oxide Semiconductor FieldEffect Transistor) external gate (gate 1 and gate 2) processed on a high resistivity silicon substrate. The DEPFET device is turned on and off by applying appropriate (external) gate-source voltage, which controls the source-drain current. Beneath the external gates of the MOSFETs, additional n-implanted regions force the generated (photo-) electrons to drift underneath the gates and confine them in an electric field. The collected electrons in these locations called "internal gate" also modulate the MOSFET channel conductivity and thus have an impact on the source-drain current, and the amount of charge is measured by the difference of the measured source-drain current before and after the collected charge is moved away from the internal gate. In DEPFET-RNDR device, the transfer gate acts as a n-channel MOSFET which couples the two internal gates, and by applying a positive voltage to the transfer gate the collected charge can be transfered from the internal gate of the on-DEPFET to the internal gate of the off-DEPFET. Because there is no loss in transferring the charge to the neighboring DEPFET, the amount of charge can be measured multiple times, therefore the effective noise scales to $1 / \sqrt{N}$ where $N$ is the number of repetitions in the readout, 
following the reduction of statistical error from the repetition of the same measurement. Further details on the realization of RNDR on DEPFET devices can be found in [6].

Measurements on the DEPFET RNDR single pixel prototypes developed and produced by HLL proved the basic functionality and the potential to reduce the effective noise. The achieved optimal noise level, however, was limited by the dark current electrons which arrive during the RNDR readout, and the limitation was mainly due to the operating temperature of $-40{ }^{\circ} \mathrm{C}[5]$ in the measurement. Previous studies showed that at a temperature of about $-70{ }^{\circ} \mathrm{C}$, we can reach the expected 0.2 ENC level for dark matter-electron scattering detection.

\subsection{DANAE status and future perspectives}

Based on the previous results, we are preparing a proof-of-principle measurement for a $64 \times$ 64 pixel matrix produced by HLL, with a total mass of $24 \mathrm{mg}$. Our main objective is a complete parameterization and characterization of the device performance as a function of temperature, using a setup shown in Fig. 1 (b). The setup can provide more cooling power than the previous measurement, and we have performed a cooling test without the detectors to confirm the performance of the cryocooler. Suitable ASICs used to control the matrix gates and clear voltages are available as well as ASICs to process and acquire the signals from the sensor. The required electronic boards implemented with the ASICs are under production. By the end of 2018, we will finish the assembly of the complete detector system and the implementation of the software for the matrix controlling and data acquisition. The performance of the prototype will be tested and optimized in early 2019. The longer term plan of introducing larger arrays for physics run is currently under discussion and its timeline will depend on the outcome of the proof-of-principle measurement.

\section{Summary}

Earlier measurements [5] [6] have verified that the DEPFET with RNDR architecture is capable to identify energy deposit in the silicon sensor that corresponds to a single electron charge. Such sensitivity enables us to look for signals from a sub-GeV/c ${ }^{2}$ mass dark matter candidate which scatters off a bound electron inside the silicon sensor. As the first step to realize this concept, we are preparing a proof-of-principle measurement to readout a prototype detector array with $24 \mathrm{mg}$ sensitive volume. We will confirm the performance of the prototype in early 2019 , and determine a future physics run accordingly.

\section{References}

[1] T. M. Undagoitia and L. Rauch, J Phys G: Nucl Part Phys 43 (1) (2016) 013001.

[2] R. Essig et al., JHEP 05 (2016) 046, [hep-ph/1509 . 01598].

[3] A. Aguilar-Arevalo et al., Phys. Rev. Lett. 118 (14) (2017) 141803.

[4] J. Tiffenberg et al., Phys. Rev. Lett. 119 (2017) 131802.

[5] A. Bähr, H. Kluck, J. Ninkovic, J. Schieck, and J. Treis, Eur. Phys. J. C 77 (2017) 905,

[6] S. Woelfel et al., IEEE Trans. Nucl. Sci. 54 (4) (2007) 1311.

[7] J. Treis et al., IEEE Trans. Nucl. Sci. 52 (4) (2005) 1083. 